\title{
Simulating Web Applications Design Models
}

\author{
Pedro Peixoto \\ Faculty of Engineering \\ University of Technology, Sydney \\ Broadway, PO Box 123 \\ Sydney, NSW 2007, Australia
}

\begin{abstract}
In the last decade several design models have been proposed and efficiently used for the developing of highly complex Web applications. Their suitability to deal with the Web design intricacies have result in a wide acceptance from the Web developer community. There has however been little consideration given to the simulation of the resulting design models. Simulation of the models would provide developers with the means for an in-depth analysis and assessment of the design, contributing for the reduction of both length and cost of the testing phase of the software life cycle. This paper presents a simulation tool that has been developed for the evaluation of Web application design models.
\end{abstract}

\section{Introduction}

Web application design has reached a point where only with specialized modeling techniques are developers able to tackle its complexity. To cope with that, several design models have been proposed and a few have gained wide acceptance for their suitability for addressing complex design issues. Design models such as UML [3], WebML [4] and OOHDM [5] are commonly used by developers in the Web application design field. However, developers still have to undertake some coding in order to test and assess specific aspects of the applications, namely presentation, navigation, functionality, and data access and content issues.

Simulation of Web design models has several notable advantages - developers may more accurately and almost instantaneously evaluate the design requirements by observing its response to a set of well known stimulus. Presently, however, simulation of the design model itself has been almost entirely neglected. This paper argues that simulating Web application design models is a not only a desirable but an attainable objective.

\section{The WDL Simulation Model}

To be able to simulate the several heterogeneous existent Web application design models with a single simulation tool, a new description language was developed - the Web Description Language (WDL) [1, 2]. Essentially, the design model written in languages such as UML or WebML, is mapped into a WDL design

D. Lowe and M. Gaedke (Eds.): ICWE 2005, LNCS 3579, pp. 627-629, 2005.

(C) Springer-Verlag Berlin Heidelberg 2005 
using four basic entities: Page, Link, Script, and Data, each encompassing a description of its structure and behavior. These basic WDL entities may be further extended and combined to form more intricate components. The resulting WDL model is then loaded by the simulator tool and the system awaits for stimuli to process and proceed.

The WDL simulation model revolves around a four layer concept, namely: Presentation, Navigation, Functional, and Content. It is from these layers perspective that simulation is performed, processed, displayed, and evaluated. A more thorough description of each layer objectives and implementation can be found in [2].



Fig. 1. A snapshot of the WDL Simulation Tool

\section{Implementation and Example}

The WDL Simulation Tool was implemented using Java and has been progressively refined to maximize its analysis capabilities. Simulation is driven by the input events which act upon the design's constituents, each of which is capable of processing the stimuli and reacting accordingly. The graphics module renders the stimulus and outcome of the simulator engine into a suitable and convenient format for a straightforward and meaningful analysis.

The simulator entails support for multi-session and multi-user processing, contributing to an even more powerful and complete analysis tool. Distinct users 
may be simultaneously simulated, each with their own private and shared data space, and concurrent access issues to shared resources such as files or databases may be promptly assessed. These features lead to significantly broaden the scope of the simulation analysis, by not only simulating the design itself but by also taking in consideration the concurrent and distributed nature of a Web application implementation.

Figure 1 shows a snapshot of part of a simulation of an online music store Web application, in which two users are simultaneously accessing the application. The simulator enables a meaningful evaluation of the impact that each user's followed path has on the application's state from the above-mentioned four layers perspective. The Web application state resulting from a specific stimulus may be observed and verification of its correctness may be easily performed.

At each instant access to the Web application state is possible, namely the content being displayed to the user, the value of the variables of the executed scripts, and the content of each data entity. This enables a more thorough, flexible and automatic testing phase, leading to a faster error identification and, consequently, to a higher quality of the final product.

\section{Future Work}

Future work will focus on improving the analysis capabilities of the simulator, such as a requirements assessment module that will automatically evaluate the Web model state looking for a set of specific conditions to be met.

\section{References}

1. Pedro Peixoto and KK Fung and David Lowe, A Framework for the Simulation of Web Applications, ICWE'04, Munich, Germany, 2004.

2. Pedro Peixoto and KK Fung and David Lowe, A Framework for the Simulation of Web Applications - Technical Report, Technical report, University of Technology, Sydney, Australia, 2004.

3. Conallen, Jim, Addison-Wesley, Building Web Applications with UML, Addison Wesley Object Technology Series, 1999

4. Ceri, S. and Fraternali, P. and Bongio, A., Web Modeling Language (WebML): a modeling language for designing Web sites, Proceedings of WWW9 Conference, Amsterdam, 2000

5. Schwabe, Daniel and Rossi, Gustavo, The Object-Oriented Hypermedia Design Model, Communications of the ACM, vol. 38, number 8, pp. 45-46, 1995

6. Ashenden, P., The Designer's Guide to VHDL, Morgan Kaufmann Publishers, 1st Ed. 2002 